\title{
Effects of heat stroke on surface ECG: a study on clinical outcomes
}

\author{
Amal Paul, ${ }^{\oplus 1}$ Reginald Alex, ${ }^{2}$ John Roshan Jacob, ${ }^{1}$ Bijesh Yadav ${ }^{3}$
}

${ }^{1}$ Cardiology and cardiac electrophysiology, Christian Medical College and Hospital Vellore, Vellore, Tamil Nadu, India

${ }^{2}$ Accident and Emergency, Christian Medical College and Hospital Vellore, Vellore, Tamil Nadu, India

${ }^{3}$ Biostatistics, Christian Medical College and Hospital Vellore, Vellore, Tamil Nadu, India

\section{Correspondence to}

Professor John Roshan Jacob, Cardiology, Christian Medical College and Hospital Vellore, Vellore 632004, Tamil Nadu, India; johnroshanjacob@gmail. com

Received 9 April 2019

Revised 5 May 2019

Accepted 22 May 2019
Check for updates

(C) Author(s) (or their employer(s)) 2019. No commercial re-use. See rights and permissions. Published by BMJ.

To cite: Paul A, Alex R, Jacob JR, et al. Heart Asia 2019;11:e011221. doi:10.1136/

heartasia-2019-011221

\section{ABSTRACT}

Aims Classic heat stroke is associated with high inhospital mortality and morbidity. The relation between the ECG findings in heat stroke and the clinical outcomes of these patients has not been studied. The aim of this study was to describe the electrocardiographic features in patients with classic heat stroke and to determine if there is any correlation of ECG findings with in-hospital outcomes.

Methods We performed a retrospective study on 50 patients with classic heat stroke during summer months of 2016-2018. All 12-lead electrocardiographic recordings obtained from these patients were subjected to in-depth analysis. Statistical analysis was done to determine the correlation of electrocardiographic findings with in-hospital outcomes.

Results 37 patients were in sinus rhythm, while supraventricular arrhythmias including atrial fibrillation $(n=6)$, ectopic atrial tachycardia $(n=4)$ and atrial flutter $(n=2)$ were observed in the rest. There was a high prevalence of QTc prolongation, low voltage P waves, conduction defects like incomplete right bundle branch block and repolarisation abnormalities. The ratio of QRS voltage in the limb leads to that in precordial leads was $\leq 0.5$ in nearly three-fourths of the patients. Among the observed electrocardiographic features, low P-wave voltage $(<0.01 \mathrm{mV})$ in lead II was found to have statistically significant correlation with adverse inhospital outcome (OR 8.93, $p=0.04$ ), after adjustment for clinical covariates.

Conclusion There was high incidence of atrial arrhythmias in patients with classic heat stroke. A low P-wave voltage $(<0.01 \mathrm{mV})$ in lead II was predictive of adverse in-hospital outcome in this cohort of patients.

\section{BACKGROUND}

Heat stroke is defined as core body temperature usually in excess of $40^{\circ} \mathrm{C}\left(104^{\circ} \mathrm{F}\right)$ with associated central nervous system dysfunction in the setting of a large environmental heat load that cannot be dissipated. ${ }^{1}$ Classic (non-exertional) heat stroke commonly affects elderly individuals with underlying chronic medical conditions that impair thermoregulation, interfere with access to hydration or attempts at cooling. Reduced epidermal area available for heat transfer, impaired cutaneous vasodilation and impaired ability to deliver heat to the skin make the elderly population more susceptible to non-exertional heat stroke. ${ }^{23}$ Exertional heat stroke generally occurs in young, otherwise healthy individuals, typically athletes and military trainees who engage in heavy exercise during periods of high ambient temperature and humidity.

Cardiac manifestations of heat stroke described in the literature range from a myriad of ECG changes ${ }^{4}$

\section{Key questions}

What is already known about this subject?

- Patients with heat stroke have a high mortality rate ranging from $21 \%$ to $63 \%$ in various cohorts. Cardiac manifestations of heat stroke described in the literature range from a myriad of ECG changes to acute heart failure, stress cardiomyopathy, acute myocardial infarction and incessant ventricular arrhythmias. The relation between the ECG findings in heat stroke and the clinical outcome of these patients has not been analysed.

\section{What does this study add?}

- There was a high incidence (24\%) of atrial arrhythmias in patients with heat stroke, which included atrial fibrillation $(n=6)$, atrial flutter $(n=2)$, ectopic atrial tachycardia $(n=3)$ and one case of multifocal atrial tachycardia, most of which were of new onset and reverted after establishing normothermia.

- The high prevalence of low voltage QRS complexes in the limb leads with relative preservation of QRS voltage in the precordial leads observed in this study has not been described in the available literature pertaining to patients with heat stroke.

- A significant proportion of the patients had no discernible P wave in lead II, owing to the extremely low voltage, suggesting the probability of significant atrial myocardial oedema.

- Low P-wave voltage $(<0.01 \mathrm{mV})$ in lead II was found to have statistically significant correlation with adverse in-hospital outcome (OR 8.93, $\mathrm{p}=0.04$ ), after adjustment for clinical covariates.

How might this impact on clinical practice?

- The novel findings described in the study add significantly to the understanding of the electrocardiographic profile in patients with hyperthermia and heat stroke. Low-voltage p wave in lead II is a potential marker of prognostication in patients with heat stroke.

to acute heart failure, stress cardiomyopathy, ${ }^{5}$ acute myocardial infarction ${ }^{6}$ and incessant ventricular arrhythmias. ${ }^{7}$ Patients with heat stroke have a high mortality rate ranging from $21 \%$ to $63 \%$ in various cohorts. ${ }^{8}$ The relation between the ECG findings in heat stroke and the clinical outcome of these patients has not been analysed. Our study was conducted in Christian Medical College and Hospital Vellore, a tertiary-care referral centre in South India, which is located 12.9 degrees north of the equator and 79.1 
degrees east of the prime meridian, $220 \mathrm{~m}$ above the mean sea level. During the months of April and May, the average ambient temperature in this part of the world is above $39^{\circ} \mathrm{C}\left(102^{\circ} \mathrm{F}\right)$ and the maximum recorded temperature is as high as $45^{\circ} \mathrm{C}\left(113^{\circ} \mathrm{F}\right)$. Heat-related injuries, including heat exhaustion and non-exertional heat stroke, are not uncommon during this period. We performed an in-depth analysis of 12-lead ECGs obtained from patients who presented with heat stroke and analysed the findings looking for markers that suggest a poor prognosis.

\section{METHODS}

This was a single-centre study with a retrospective observational design, conducted among patients who presented to the department of emergency medicine in the summer months (April, May) between 2016 and 2018, with hyperthermia (core temperature $\geq 40^{\circ} \mathrm{C}$ or $104^{\circ} \mathrm{F}$ ), altered mental status and an admission diagnosis of non-exertional heat stroke. Patients whose hyperthermia and altered mentation were unequivocally proven to be secondary to alternative aetiologies such as infection were not included in the study. Patients were included in the study only if the earliest available ECG was taken either at the time of presentation or within an hour of institution of cooling measures, so that the ECG characteristics could be presumed to be representative of high core temperature. The study was approved by the institutional review board and ethics committee.

Eighty-five patients with an admission diagnosis (in the emergency department) of possible heat stroke were identified during the study period, some of whom were eventually diagnosed to have infections affecting the central nervous system including meningitis, Falciparum malaria and enteric fever. Among the 72 patients in whom heat stroke was confirmed to be the final diagnosis, 22 were excluded because the ECG was either not available or the first available ECG was taken much later than the time of return to normothermia. Thus, a total of 50 patients were included in the study.

The clinical data, biochemical parameters, ECGs and the details of the in-hospital course until the time of discharge were obtained from the electronic medical records. All documented ECGs during the index admission were subjected to detailed analysis by two different cardiologists. Details including demographic profile, comorbidities, admission temperature, course in the hospital, duration of hospital stay and discharge status were documented. Acquired data were summarised using mean with SD for continuous variables and percentage for categorical variables. Continuous variables were compared using independent t-test and categorical variables with $\chi^{2}$ test. Statistical analysis of the data was done using SPSS V.25 software.

\section{RESULTS}

\section{Clinical profile}

The mean age of the study population was $60 \pm 12$ years (range, 29-84), of which $68 \%$ were men. The mean core temperature at the time of presentation was $105^{\circ} \mathrm{F}$ or $40.5^{\circ} \mathrm{C}$ (range, $104-109^{\circ} \mathrm{F}$ or $40-42.7^{\circ} \mathrm{C}$ ). The mortality rate was $16 \%$ and the average duration of hospital stay was $6.5 \pm 5$ days. Fifty-eight per cent of the patients developed multiorgan dysfunction. The baseline characteristics of the study population are depicted in table 1 .

Careful review of drug history revealed that one patient was on phenothiazine and another was on amitryptiline. However, there were no ECG changes attributable to these drugs. Although mild derangements in serum electrolytes were noted,

\begin{tabular}{ll}
\hline Table 1 Baseline clinical profile & \\
\hline Age (mean) & $60 \pm 12$ years \\
\hline Sex & \\
\hline \multicolumn{1}{l}{ Males } & $68 \%$ \\
\hline Females & $32 \%$ \\
\hline Temperature & $105^{\circ} \mathrm{F}$ or $40.5 \pm 1.5^{\circ} \mathrm{C}$ (range, \\
& $\left.40-42.7^{\circ} \mathrm{C} / 104-109^{\circ} \mathrm{F}\right)$ \\
\hline Diabetes mellitus & $54.3 \%$ \\
\hline Hypertension & $45.7 \%$ \\
\hline Ischaemic heart disease & $26.7 \%$ \\
\hline Creatinine phosphokinase & $3566 \pm 7102$ (range, 17-37 270) U/L \\
\hline Troponin T & $145 \pm 147$ (range, 9.1-529.4) $\mathrm{pg} / \mathrm{mL}$ \\
\hline Multiorgan dysfunction & $58 \%$ \\
\hline Duration of hospital stay & $6.5 \pm 5($ range, $0-18)$ days \\
\hline Outcomes & \\
\hline Discharged in healthy state & $64 \%$ \\
\hline In-hospital death & $16 \%$ \\
\hline Discharged against medical advice & $20 \%$ \\
\hline
\end{tabular}

case-to-case analysis of ECGs did not suggest any correlation of ECG changes with dyselectrolytaemia.

\section{Electrocardiographic profile}

Rhythm and axis

Majority of the patients $(74 \%, n=37)$ were in sinus rhythm at the time of presentation, of which two patients had first-degree atrioventricular block. The mean heart rate was $117 \pm 35$ (range, 72-218) beats per minute. Four patients had ectopic atrial tachycardia, of which one had multifocal atrial tachycardia.

Six patients were in atrial fibrillation at the time of presentation, two reverted spontaneously to sinus rhythm, while two were cardioverted-one with DC shock and the other with intravenous amiodarone. Two patients continued to be in atrial fibrillation. The mean QRS axis was normal in $78 \%$ of the patients $(\mathrm{n}=39)$. The distribution of various rhythm abnormalities and mean QRS axis at presentation are depicted in figure 1.

ECG intervals, durations and voltages are shown in table 2.

Two patients had prolongation of PR interval. The mean corrected QT interval (QTc) was $471 \mathrm{~ms}$ and the median was 465 $\mathrm{ms}$, both at the upper limit of normal. Eight patients (16\%) had right bundle branch block (RBBB), of which six had incomplete RBBB and two had complete RBBB. There was no left bundle branch block in any of the ECGs. Non-specific intraventricular conduction delay (IVCD) was noted in four patients.

It was noticed that the P-wave and QRS complex voltages were low among the ECGs studied. Forty per cent of patients $(n=20)$ had QRS voltages $\leq 0.5 \mathrm{mV}$ in all their limb leads. In contrast, however, the precordial lead QRS voltage (calculated as the sum of the maximum S-wave voltage in V1/2 and the maximum R-wave voltage in V5/6) was low $(\leq 1 \mathrm{mV})$ in just $18 \%(\mathrm{n}=9)$ of the patients. R-wave voltage in lead V3 was $\geq 3 \mathrm{~mm}$ in majority of the patients $(n=42)$. This suggested that there existed a discordance in the QRS voltages between limb and precordial leads in these patients. In order to objectively assess the significance of this finding, we calculated the ratio of maximum QRS voltages between the limb leads and the precordial leads. The QRS voltage in any given lead was measured by taking the peak to trough QRS amplitude. The ratio obtained by dividing the highest QRS voltage from among the limb leads with that from the precordial leads was $\leq 0.5$ in $74 \%$ of the patients studied $(n=37)$. This further corroborated the observation that the limb 


\section{Rhythm}

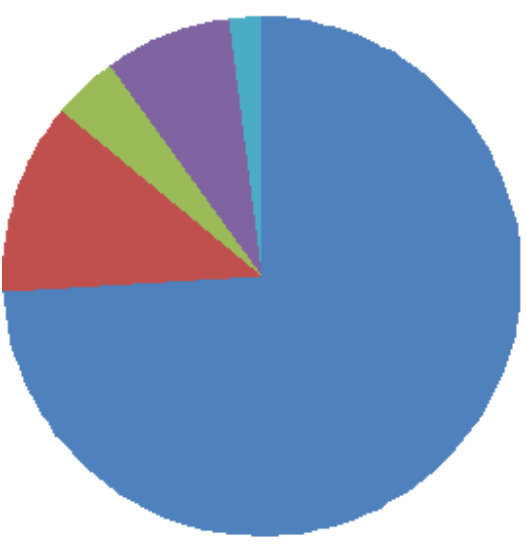

Mean QRS axis

Sinus rhythm
$[n=37]$
Atrial fibrillation
$[n=6]$
Atrial flutter
$[n=2]$
Ectopic atrial
tachycardia $[n=4]$
Junctional
rhythm $[n=1]$

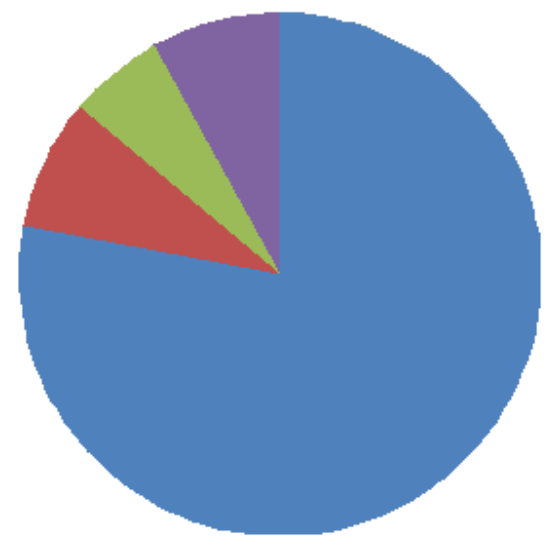

Normal $[n=39]$

Left axis deviation[n=4]

Right axis deviation $[n=3]$

Extreme right axis $[n=4]$

Figure 1 Rhythm abnormalities (left) and QRS axes (right) at presentation.

lead QRS voltages were much lower than those of the precordial leads among these patients (figure 2). Fifteen (30\%) patients had a deep Q wave in lead III.

P-wave voltage, as measured in lead II, was found to be $\leq 0.01$ $\mathrm{mV}$ in $74 \%$ of the patients who had evidence of atrial depolarisation. The P-wave voltage in lead V1 was $\leq 0.01 \mathrm{mV}$ in $81 \%$ of the patients, suggesting that the P-wave voltage is generally low across both limb and precordial leads in these patients.

\section{Repolarisation abnormalities}

Repolarisation abnormalities like ST depression and T inversion were noted in the study group, with involvement of lateral, inferior and anteroseptal leads seen in six, five and three patients, respectively. Two patients had diffuse changes not corresponding to a single coronary artery territory. Isolated $\mathrm{J}$ point elevation was seen in the anterior leads in six patients. True ST segment elevation was not seen in any of the ECGs.

None of the ECG findings were found to have any significant correlation with the absolute core temperature recorded at the time of admission to the emergency department.

\begin{tabular}{ll}
\hline Table 2 & ECG intervals, durations and voltages \\
\hline PR interval (ms) & $144 \pm 28$ (range, 100-220) \\
\hline QTc (corrected QT interval) (ms) & $471 \pm 70$ (range, 321-654) \\
\hline QRS duration (ms) & $94 \pm 19$ (range, 62-160) \\
\hline QRS voltage (mV) & \\
\hline Limb lead (maximum) & $0.7 \pm 0.3$ (range, 0.2-1.5) \\
\hline Precordial lead (sV1/2+rV5/6) & $1.8 \pm 0.8$ (range, 0.5-3.5) \\
\hline Ratio of limb/precordial lead voltages & $0.47 \pm 0.43$ (range, 0.11-3) \\
\hline R progression (R wave amplitude in V3) (mV) & $0.7 \pm 0.5$ (range, 0.15-3) \\
\hline P-wave voltage (mV) & \\
\hline Lead II & $0.01 \pm 0.006$ (range, 0.005-0.03) \\
\hline Lead V1 & $0.01 \pm 0.004$ (range, 0.005-0.02) \\
\hline
\end{tabular}

\section{Electrocardiographic changes and the clinical outcomes}

Statistical analysis was done to evaluate the correlation between the salient electrocardiographic findings and the clinical outcomes of the patients. The outcome variables considered were in-hospital mortality, duration of hospital stay and multiorgan dysfunction. While considering in-hospital mortality as an outcome, the patients who were discharged against medical advice in an unstable state were considered to have had an adverse outcome and hence were considered alongside those patients who succumbed to their illness in hospital.

Among all the electrocardiographic features described, low P-wave voltage $(<0.01 \mathrm{mV})$ in lead II was found to have a significant correlation with adverse in-hospital outcome. Multivariate analysis was done to adjust for clinical covariates such as age, comorbid illnesses, hypotension at presentation and serum creatinine phosphokinase levels. The correlation of low P-wave voltage in lead II with adverse in-hospital outcome was found to be statistically significant after adjustment for clinical covariates (OR 8.93, 95\% CI 1.1 to $76.7, \mathrm{p}=0.04$ ). Hypotension at presentation was also found to adversely affect the clinical outcome (OR 17.8, 95\% CI 2.5 to $127.2, \mathrm{p}=0.004$ ).

\section{DISCUSSION}

Electrocardiographic findings described in patients with heat-related illness include sinus tachycardia, conduction defects, prolonged QT interval, diffuse ST-T changes, as well as ST-T changes localised to the distribution of a coronary artery. ${ }^{9}$ Although earlier studies had attributed most of the ECG changes to electrolyte imbalances accompanying heat stroke, subsequent studies done in a larger number of patients have failed to find any consistent correlation in this regard. ${ }^{9}$ The salient ECG findings noted in our study including low P-wave voltage, supraventricular arrhythmias and prolonged QT interval were confirmed to be not attributable to underlying dyselectrolytaemia or background drug therapy. 


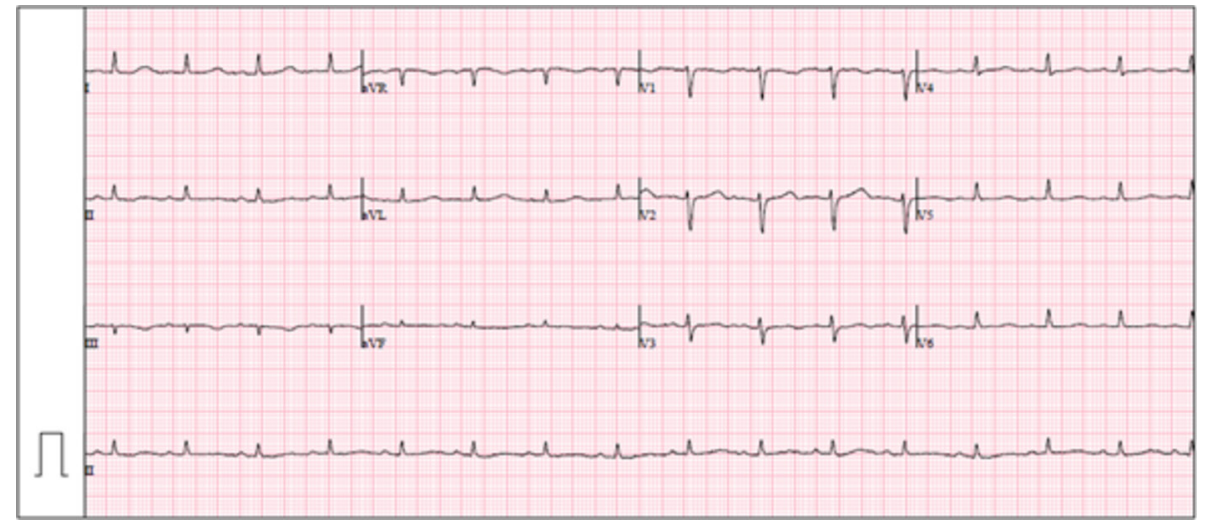

Figure 2 ECG showing low voltage QRS complexes during heat stroke.

Our study demonstrates a high incidence (24\%) of atrial arrhythmias in the study population, which includes atrial fibrillation $(n=6)$, atrial flutter $(n=2)$, ectopic atrial tachycardia $(n=3)$ and one case of multifocal atrial tachycardia, in addition to sinus tachycardia which was noted in 17 patients. The fact that these arrhythmias were documented for the first time in the respective patients and did not recur after achieving normothermia in most of them suggests that hyperthermia or the other pathophysiological processes accompanying heat stroke have served as the precipitating factors.

A high incidence of conduction defects including non-specific intraventricular conduction delay have been reported in earlier studies, which have been attributed to multiple factors including a change in the conduction rate of the ramifications of the right bundle branch due to the possible role of heat shock proteins in ion channel trafficking in the cell membrane and increased pressure in the right ventricle (resulting in incomplete RBBB). ${ }^{10}$ The incidence of conduction defects in our study cohort was 24\%, which included complete RBBB, incomplete RBBB and non-specific IVCD. Notably, LBBB was not seen in any of the patients studied.

In total, $48 \%$ of the study population had prolongation of corrected QT interval at presentation (the upper limit of normal being taken as $460 \mathrm{~ms}$ for men and $470 \mathrm{~ms}$ for women). However, this was not associated with an increased incidence of ventricular arrhythmias or mortality. This could suggest that the transient prolongation precipitated by hyperthermia resolves once normothermia is achieved and hence does not exert a lasting effect on the clinical outcome of the patient.

One of the most striking findings in our study was the low-voltage QRS complexes in the limb leads with relative preservation of QRS voltages in the precordial leads, which has hitherto not been described in this cohort. The ratio of maximal limb to precordial QRS voltage was 0.5 or less in nearly threefourths of the patients studied. Although the distance of the limb leads from the heart is greater than the chest leads, this does not usually contribute much to a difference in magnitude of the recorded complexes with modern electrocardiographic machines. $^{11}$

Low QRS voltage in the limb leads with preservation of the precordial lead voltage (called voltage discordance) has been described in the literature as a finding consistent with infiltrative cardiomyopathy, pericardial effusion, large pleural effusions, chronic obstructive pulmonary disease and obesity. ${ }^{12}$ In patients with no pathological variation in voltage generation or transmission, this finding may be reflective of a posteriorly directed QRS axis. ${ }^{12} 13$
An association of this ECG finding with severe dilated cardiomyopathy has also been described. Ventricular dilatation causes a shift in the orientation of the mean QRS vector more in parallel with the transverse plane and perpendicular relative to the frontal plane, thereby effecting a reduction in the limb lead QRS voltage. ${ }^{14}$ Increased proximity of a dilated heart to the chest wall would also tend to increase precordial lead voltage more than the limb leads. ${ }^{12}$ These factors could possibly explain the voltage discordance in some patients with heat stroke, as ventricular dilatation secondary to myocardial dysfunction has been described in heat stroke. ${ }^{5}$

Previous studies have demonstrated that small or trivial pericardial effusions are associated with low voltages as well as voltage discordance in ECG in patients with no other identifiable explanation for the low voltages. ${ }^{12} 15$ Pericardial inflammation has been proposed as a possible mechanism for the attenuation of voltages apart from enhanced conduction by a layer of fluid. ${ }^{16}$ Echocardiographic assessment has shown pericardial effusion in as many as $25 \%$ of patients with heat stroke. ${ }^{17}$ Taken together, small pericardial effusions may be the reason for voltage discordance in a significant proportion of patients with heat stroke with no myocardial dysfunction or ventricular dilatation.

Although a universally accepted definition for lower limit of P-wave voltage is not available, the finding of low amplitude P wave across limb leads and precordial leads in this study population is worthy of discussion. About three-fourths of the study cohort had a P-wave voltage less than or equal to $0.01 \mathrm{mV}$. More importantly, a low P-wave voltage in lead II $(<0.01 \mathrm{mV})$ corresponded with a significantly higher incidence of adverse in-hospital outcome, further suggesting that the implications of this ECG finding extend far beyond the effects of mere hyperthermia and probably reflect the overall severity of the illness in such patients. The absence of a discernible P wave in lead II, owing to the extremely low voltage, suggests the possibility of significant atrial myocardial oedema due to the disease process. This along with the low-voltage QRS could imply global myocardial involvement.

ST-T changes suggestive of myocardial ischaemia, including segmental as well as diffuse ST elevation, ST depression and deep $\mathrm{T}$ inversion, have been described in various studies done in patients with heat stroke. Echocardiography done in patients with ischaemic ST-T changes has shown contrasting results. ${ }^{17}$ In our study, the prevalence of electrocardiographically significant repolarisation abnormalities was $28 \%(n=14)$, of which 12 were attributable to probable myocardial ischaemia. Two patients were found to have clinical and echocardiographic features suggestive of myocarditis. The ischaemic changes in ECG can probably be 
attributed to secondary myocardial ischaemia resulting from the increase in myocardial oxygen demand due to hyperthermia, tachycardia, high cardiac output state or hypotension. ${ }^{18} 19$ Diffuse vasospasm secondary to endothelial damage and catecholamine toxicity simulating stress cardiomyopathy are other potential explanations for ischaemic changes in ECG. It has also been suggested that changes in blood composition due to heat stress such as increase in haematocrit, whole blood viscosity and plasma cholesterol could account for coronary arterial thrombosis in heat stroke. ${ }^{20}$

In our study, the highly sensitive troponin $\mathrm{T}$ levels (normal $<14 \mathrm{pg} / \mathrm{mL}$ ) were more than the upper limit of normal in 19 out of the 23 patients, in whom the levels were checked. In a clinicopathological study of 125 heat stroke cases, right ventricular dilatation and left ventricular subendocardial haemorrhage with severe degenerative changes in the cardiac muscle were described. ${ }^{21}$ This could explain the elevated troponin levels in those patients with no other evidence of acute myocardial ischaemia.

Our study was limited by its retrospective design and the lack of appropriately timed serial ECGs in all patients. Although it is possible that certain findings such as incomplete RBBB may have been present in a few patients prior to the heat stroke, transient findings such as new-onset arrhythmias and the described voltage abnormalities are more likely to be a consequence of heat stroke. Most of these changes were found to have reverted in the subsequent ECGs, wherever available. Nonetheless, a baseline ECG before the heat stroke and appropriately timed serial ECGs could have contributed significantly to the understanding of the evolution of the electrocardiographic picture in this cohort.

Since heat stroke entails a more complicated pathophysiological process, the salient ECG findings noted in the study cannot be considered to be the electrophysiological attributes of hyperthermia alone. Further research is warranted to decipher whether isolated hyperthermia can bring about similar electrocardiographic changes.

\section{CONCLUSION}

Our study demonstrates a high incidence of atrial arrhythmias in patients with classic heat stroke. There was a high prevalence of low-voltage P waves, low limb lead QRS voltage with preserved precordial lead voltage, QTc prolongation, conduction defects like incomplete RBBB and repolarisation abnormalities. Low P-wave voltage $(<0.01 \mathrm{mV})$ in lead II was found to have statistically significant correlation with adverse in-hospital outcome.

Contributors AP was involved in the conceptualisation; data curation; formal analysis; investigation; methodology; roles/writing —original draft; writing—review and editing. RA was involved in the investigation; methodology; writing-review and editing. JRJ was involved in the data curation; formal analysis; investigation; methodology; roles/writing —original draft; writing—review and editing. BY was involved in the statistical analysis.

Funding The authors have not declared a specific grant for this research from any funding agency in the public, commercial or not-for-profit sectors.

Competing interests None declared.

Patient consent for publication Not required.

Ethics approval The study was approved by the institutional review board and ethics committee of Christian Medical College Vellore, Tamil Nadu, India.

Provenance and peer review Not commissioned; externally peer reviewed.

Data availability statement All data relevant to the study are included in the article or uploaded as online supplementary information.

\section{REFERENCES}

1. Bouchama A, Knochel JP. Heat stroke. N Eng/ J Med 1978;2002.

2. Bross MH, Nash BT, Carlton FB. Heat emergencies. Am Fam Physician 1994;50.

3. Klenk J, Becker C, Rapp K. Heat-related mortality in residents of nursing homes. Age Ageing 2010;39:245-52.

4. Wakino S, Hori S, Mimura T, et al. A case of severe heat stroke with abnormal cardiac findings. Int Heart J 2005;46:543-50.

5. Chen W-T, Lin C-H, Hsieh M-H, et al. Stress-induced cardiomyopathy caused by heat stroke. Ann Emerg Med 2012:60:63-6.

6. García-Rubira JC, Aguilar J, Romero D. Acute myocardial infarction in a young man after heat exhaustion. Int I Cardiol 1995;47:297-300.

7. Shimada M, Tsai B, Marshall JP. A case of heat stroke complicated by persistent ventricular tachycardia. J Emerg Med 2014.

8. Misset B, De Jonghe B, Bastuji-Garin S, et al. Mortality of patients with heatstroke admitted to intensive care units during the 2003 heat wave in France: a national multiple-center risk-factor study. Crit Care Med 2006;34:1087-92.

9. Akhtar MJ, Al-Nozha M, Al-Harthi S, et al. Electrocardiographic abnormalities in patients with heat stroke. Chest 1993;104:411-4.

10. Mimish L. Electrocardiographic findings in heat stroke and exhaustion: a study on Makkah pilgrims. I Saudi Heart Assoc 2012;24:35-9.

11. Berson AS, Pipberger HV. Skin-electrode impedance problems in electrocardiography. Am Heart J 1968;76:514-25.

12. Chinitz JS, Cooper JM, Verdino RJ. Electrocardiogram voltage discordance: interpretation of low QRS voltage only in the limb leads. J Electrocardiol 2008:41:281-6.

13. Goldberger AL, Dresselhaus T, Bhargava V. Dilated cardiomyopathy: utility of the transverse: frontal plane QRS voltage ratio. J Electrocardiol 1985;18:35-40.

14. Horan LG, Flowers NC, Thomas JR, et al. The spatial vectorcardiogram in idiopathic cardiomyopathy. Prog Cardiovasc Dis 1964;7:115-24.

15. Kamath SA, Meo Neto JdeP, Canham RM, et al. Low voltage on the electrocardiogram is a marker of disease severity and a risk factor for adverse outcomes in patients with heart failure due to systolic dysfunction. Am Heart J 2006;152:355-61.

16. Bruch C, Schmermund A, Dagres N, et al. Changes in QRS voltage in cardiac tamponade and pericardial effusion: reversibility after pericardiocentesis and after anti-inflammatory drug treatment. J Am Coll Cardiol 2001;38:219-26.

17. al-Harthi SS, Nouh MS, al-Arfaj H, et al. Non-invasive evaluation of cardiac abnormalities in heat stroke pilgrims. Int J Cardiol 1992;37:151-4.

18. Tintinalli JE. Heat stroke. JACEP 1976:5:525-8.

19. Al-Harthi SS, Sharaf El-Deane MS, Akhtar J, et al. Hemodynamic changes and intravascular hydration state in heat stroke. Ann Saudi Med 1989:9:378-83.

20. Keatinge WR, Coleshaw SRK, Easton JC, et al. Increased platelet and red cell counts, blood viscosity, and plasma cholesterol levels during heat stress, and mortality from coronary and cerebral thrombosis. Am J Med 1986;81:795-800.

21. Malamud N, Haymaker W, Luster RF, et al. A clinico-pathological study of 125 fatal cases. Milit Surg 1946;99:397-449. 